\title{
Human hand modelling: kinematics, dynamics, applications
}

\author{
Agneta Gustus • Georg Stillfried • Judith Visser • \\ Henrik Jörntell · Patrick van der Smagt
}

Received: 1 March 2012 / Accepted: 15 October 2012 / Published online: 7 November 2012

(C) The Author(s) 2012. This article is published with open access at Springerlink.com

\begin{abstract}
An overview of mathematical modelling of the human hand is given. We consider hand models from a specific background: rather than studying hands for surgical or similar goals, we target at providing a set of tools with which human grasping and manipulation capabilities can be studied, and hand functionality can be described. We do this by investigating the human hand at various levels: (1) at the level of kinematics, focussing on the movement of the bones of the hand, not taking corresponding forces into account; (2) at the musculotendon structure, i.e. by looking at the part of the hand generating the forces and thus inducing the motion; and (3) at the combination of the two, resulting in hand dynam-
\end{abstract}

This article forms part of a special issue of Biological Cybernetics entitled "Multimodal and Sensorimotor Bionics".

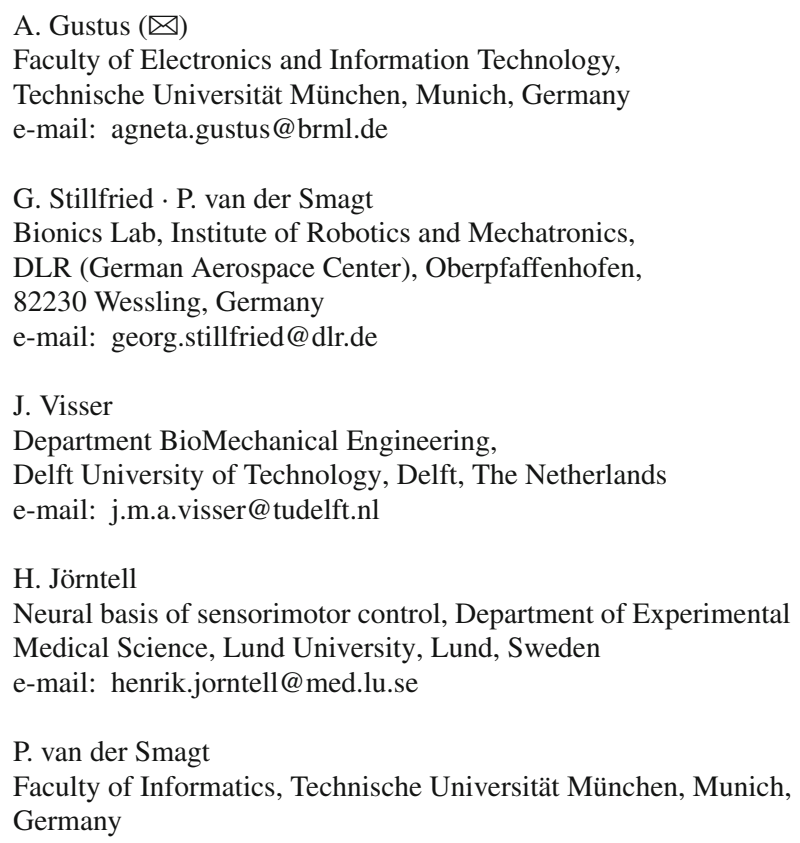

Germany

ics as well as the underlying neurocontrol. Our purpose is to not only provide the reader with an overview of current human hand modelling approaches but also to fill the gaps with recent results and data, thus allowing for an encompassing picture.

Keywords Human hand model · Hand kinematics . Muscle dynamics $\cdot$ Tendon dynamics $\cdot$ Cadaver studies

\section{Introduction}

The human hand is one of the environmental perception organs and the manipulation organ at our disposal. owing to the development of the upright locomotion, the hand was free to develop from the foot-hand (Napier 1993) to the highly skilled manipulative hand of modern man. It has become the contact tool to our environment; with it, we have shaped our surroundings for thousands of years. Firstly, we built tools and created objects for daily use. As time passed, our impact upon nature and life on the earth increased.

We have a large number of tools specially made for us to deal with our environment. Just think about your mug or your mobile phone. Even your shoes are a product of our hands.

You will come to the conclusion that, without the development of the purely manipulative hand, human development would not have become further than that of monkeys. For that evolution, the development of upright locomotion and a simultaneous increase of brain size are crucial. It is all a product of brain and hand, the cognitive possibility of tool use and the ability of the tool.

It therefore makes sense that robotic grippers, when used in the human environment, should be designed based upon the function of human hands. They may look different, of course, but should have similar gripping and manipulation 
capabilities, a comparable size, etc. Since the 1960s, robotic gripper designs have increasingly mimicked the human hand in efforts to obtain a manipulating tool for robots. The aim is not to copy human grasping and manipulation, but to design a tool for robots which can adequately deal with the human-shaped environment and the tasks within that environment. Early developments were born out of prosthetics needs; for instance, the Vaduz hand in 1949 (Wilms and Nader 1951) and the Otto Bock hand in 1965 (Marguardt 1965) were some of the first electrically driven functional hand prostheses. Later, more complex robotic hands emerged. Still such developments pose a trade-off in the challenge of increasing dexterity, while decreasing weight and size; a path usually chosen is that of building tendon-driven hands, where the actuator can be more proximal from the fingers. The first tendon-driven robotic hands included the three-fingered Stanford/JPL (Stanford University/NASA Jet Propulsion Laboratory) hand in 1985 (Salisbury 1988, 1991) and the four-fingered MIT/Utah (Massachusetts Institute of Technology/Utah University) hand in 1986 (Jacobsen et al. 1986). More recently, the German Aerospace Center (DLR) developed a tendon-driven hand (Grebenstein and van der Smagt 2008; Grebenstein et al. 2011), kinematically shaped after the human hand. This hand has a theoretically larger configuration space than the human hand because of its independent joint architecture; each joint of the 19-DoF hand is controlled by two independent motors.

When faced with such mechatronic possibilities, the question arises whether such an independent architecture is advantageous from a grasping and manipulation point of view. To understand this question and how to answer it, we want to understand grasping and manipulation, and try to understand this based on detailed human hand models.

Most grasping tasks can be categorised in two basic grips: Power grip and pinch grip. In power grip, the object is in the palm of the hand and enclosed by the fingers; in pinch grip, the object is held between the tip of the thumb and finger. Power grip is inherently stable since the finger orientation is prescribed and constrained by the object held. Pinch grip requires the 6 joints between the index finger and the thumb to be stabilised; it requires more activity of the intrinsic finger muscles to maintain this balance.

After that, more complicated tasks such as manipulating objects can be investigated. Based on that knowledge, a set of abilities for different robot hands, like in medical environments or assistive robots in home environments, can be derived.

But, up to what extent are these functionalities a result of the dexterity of our hands, and how much is grasping limited by our hands? Which part of the functionality must be copied in robotic grippers, and which part is only a 'byproduct' of our biophysics? To answer these questions, we need models of the human hand with which the grasp functionality can be simulated. By separating the kinematic properties, involving the position of the joints, from the dynamics and actuation, involving the movement, we can separately investigate the kinematic and dynamic restrictions and useful properties of our hands.

Our first step therefore is to obtain data about human hand kinematics (Stillfried and van der Smagt 2010; Youm et al. 1978). Second, human hand actuation has to be investigated. With this information, a functional description of human hand movement and force generation can be obtained.

A recent research trend has been the reduction of complexity of hand control by synergies. In its basic meaning, a synergy defines relationships between different joint angles in the hand and can thus be used to control a whole-hand movement by a single variable. By designing the optimal shape of a finger, even an underactuated hand can have a stable grasp for a range of objects (Kragten and Herder 2010). Underactuated robot hands as developed at TU Delft are able to grasp a vast range of objects with minimal activation due to the coupling of the joints (Kragten et al. 2011).

In this prospective paper, we want to focus mainly on the issues of human hand modelling from kinematic, muscular-tendon and dynamic point of view. Some insight on neurological control of human hand function will be given.

\section{Kinematic models}

Kinematics is the study of motion without regarding the forces which cause the motion. In the context of hand modelling, it refers to a set of possible motions which a hand can do. Generally, kinematics deals with positions, velocities and accelerations, orientations, angular velocities and angular accelerations. Here, we mainly study positions and orientations (the system is considered to be quasi-static).

While many studies on human hands include the wrist, in this section we take the robotics point of view: the arm is responsible for the pose of the end-effector (gripper, hand), and the end-effector is responsible for the interaction with objects. This makes the wrist a part of the arm, leaving the palm, thumb and fingers to the hand. The palm consists of four metacarpal (MC) bones, the fingers 2 to 5 consist of three bones each (proximal (PP), middle (MP) and distal (DP) phalanx) and the thumb consists of three bones (MC, PP and DP) (Fig. 1). The bones are regarded as rigid segments in technical terms so the state of the quasi-static system is described by the pose (position and orientation) of each bone. The set of poses shall be called posture. In 3-D space, six parameters are needed to define the pose of a rigid object, totalling 114 parameters for the posture-if no constraints are put on each bone's pose. The goal of our kinematic modelling is the identification of the constraints on the poses of the bones. Ideally, the resulting model describes the space of all possible postures that the hand can take on. 


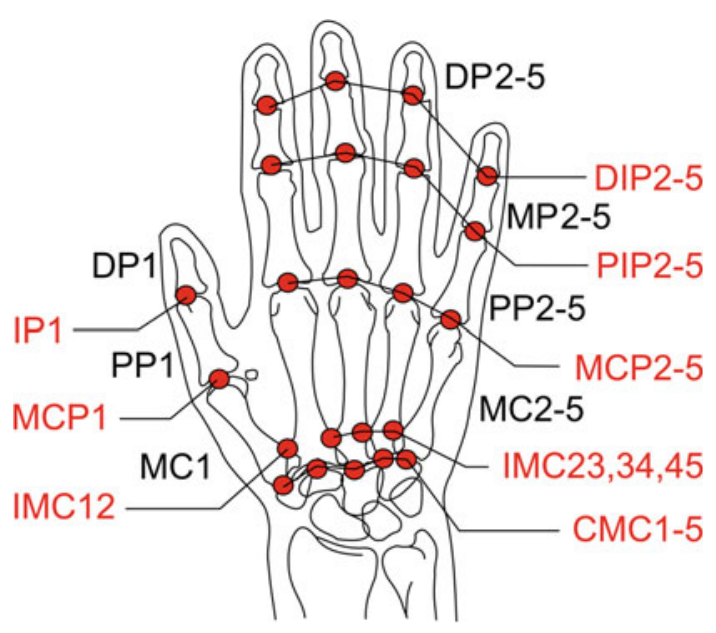

Fig. 1 The bones of a human hand consist of the carpal bones, the metacarpal (MC) bones, the proximal phalanges (PP), medial phalanges (MP) and distal phalanges (DP). They are connected by the carpometacarpal (CMC) joints, the intermetacarpal (IMC) joints, the metacarpophalangeal (MCP) joints, the proximal interphalangeal (PIP) joints, the distal interphalanges (DIP) joints and the interphalangeal (IP) joint of the thumb. The fingers are numbered as follows: 1 thumb, 2 index, 3 middle, 4 ring, 5 little

The constraints are commonly called joints. The names for the joints are metacarpophalangeal joint (MCP) for the joint which constrains the relative pose of the PP with respect to the MC, proximal interphalangeal joint (PIP) for the joint that constrains the MP pose to the PP, distal interphalangeal joint (DIP) for the joint that constrains the DP pose to the MP and interphalangeal joint for the joint that constrains the pose of DP to the PP in the thumb. Constraints of the poses of the MC to the carpal bones (=wrist bones) are called carpometacarpal joints (CMC), whereas constraints between $\mathrm{MC}$ poses are called intermetacarpal joints (IMC) (Fig. 1).

We do not consider the motion of the wrist. So, considering both CMC and IMC joints for each MC leads to a parallel kinematic structure. As those are often more complicated to deal with than serial structures, we simplify the structure to a branching serial kinematic chain: We connect the MCs through IMC joints and arbitrarily set the index finger MC as the origin of the branching kinematic chain of the hand. From there, the subchains for the thumb, index finger and middle finger start. The kinematic subchain for the ring finger branches off at the middle finger $\mathrm{MC}$, and the one for the little finger branches off at the ring finger MC.

Simple joints can be modelled using a fixed axis of rotation. This is for example done in a work by Chao et al. (1976), where the position of the axis of rotation is estimated as the centre of curvature of the bone, as seen on X-ray images. Modelling with fixed rotation axes is very common, but a few more complex models consider the pose of the rotation axis in dependence of the joint angles [e.g. van Nierop et al. (2008) model a switch from one axis position to another at a certain joint angle; Leardini et al. (1999) model a foot joint as a four-bar linkage, which results in a moving instantaneous axis of rotation].

In order to arrive at a kinematic model, the space of possible postures is sampled and interpolated. There are different methods for measuring the bone poses, invasive and noninvasive. Invasive methods involve penetrating the skin to directly measure the bone motion, e.g. by mechanical devices (Hollister et al. 1992) or optical methods (Youm et al. 1978). These methods are commonly carried out in vitro (on cadaver specimens). In non-invasive methods, either the motion of the skin is measured by optical methods (Cerveri et al. 2005) or the motion of the bones is measured by medical imaging (Stillfried and van der Smagt 2010).

Measuring skin motion introduces a soft tissue artefact (STA) on the measured bone motion due to the relative motion between skin and bone (Ryu et al. 2003). There are methods that try to model and compensate the STA (Zhang et al. 2003; Dexmart 2009). Of the medical imaging methods, magnetic resonance imaging (MRI), if used correctly, can be considered non-invasive (Dempsey et al. 2002). Methods with ionising radiation, especially computing tomography (CT), are somewhere between invasive and non-invasive as they lead to a slight but significant increase in the risk of cancer (Smith-Bindman et al. 2009).

\subsection{Optical tracking}

In optical surface marker tracking, retro-reflective markers or active markers are attached to the skin. They can be placed on the joints (Cerveri et al. 2007; Metcalf et al. 2008; Zhang et al. 2003; Choi et al. 2008) or between the joints (Cerveri et al. 2007; Chang and Pollard 2007b; Miyata et al. 2004).

\subsubsection{STA}

In an experiment, we measured the motion of the skin with respect to the bone. For this, we attached MRI-sensitive Soledum oil capsules (Casella-med, Cologne, Germany; spheroids with diameter $7 \mathrm{~mm}$ and long axis $10 \mathrm{~mm}$ ) to the skin of the hand. The capsules were centered over the joints and over the middle of the segments (Fig. 2 top). We recorded MRI images of 20 different hand postures (Table 1).

The position of each skin marker was measured as the mean of the coordinates of the capsule volume weighted by the intensity values:

$\mathbf{p}_{m} m=\frac{1}{\sum_{i \in \mathcal{V}} g_{i}} \sum_{i \in \mathcal{V}} g_{i} \mathbf{x}_{i}$,

where $\left(\mathbf{p}_{\mathrm{m}}\right)_{3 \times 1}$ is the resulting vector containing the position coordinates of the skin marker, $g_{i}$ is the intensity value (=grey value) at the $i$-th volume element of the capsule volume $\mathcal{V}$ and $\left(\mathbf{x}_{i}\right)_{3 \times 1}$ contains the coordinates of the $i$-th 


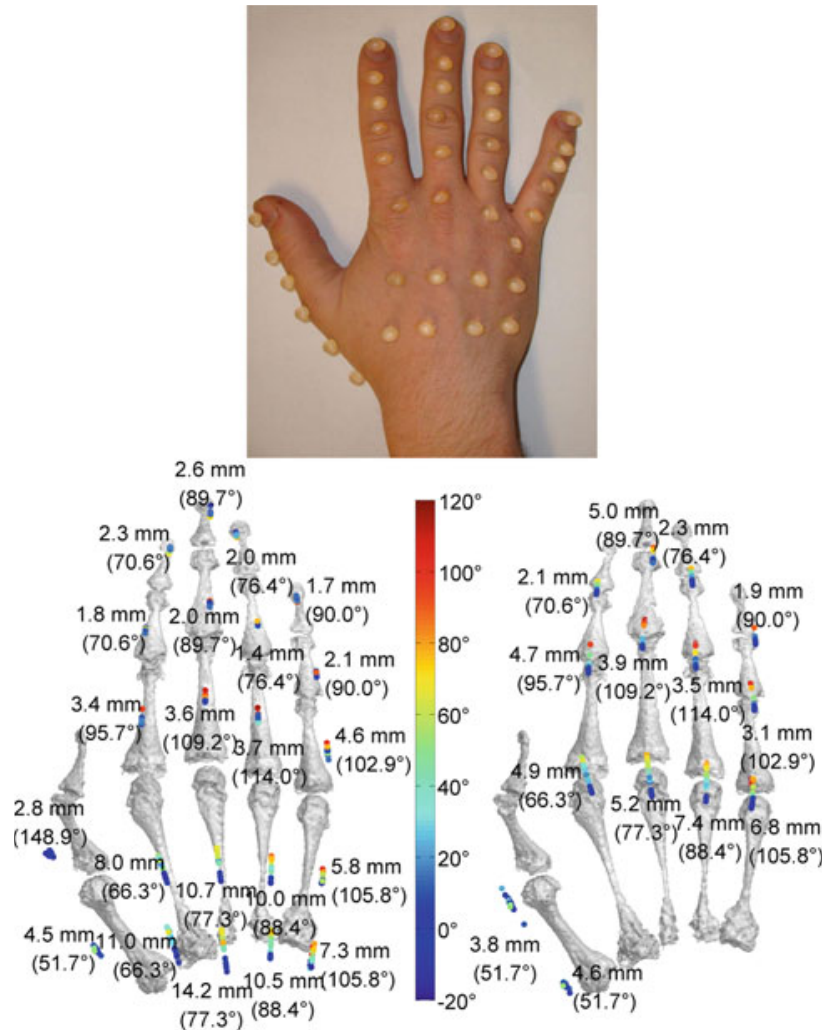

Fig. 2 Measuring soft tissue artefacts (STA). Top the movement of the skin over the bone is measured by taking MRI images with oil capsules attached to the skin. Bottom movement of the oil capsule centroid with respect to the bone in proximal-distal and palmar-dorsal direction. The movement of markers near joints is shown with respect to the bone proximal to the joint (except the marker near CMC1, the movement of which is shown with respect to the bone distal to the joint). The relative marker positions are shown in a colour indicating the flexion angle of the associated joint. Bottom left markers on the fingernails, on the bones between the joints and on the back of the hand. The amount of STA indicated near the marker centroids is the maximum distance that the marker moved from a flat-hand reference posture (in parentheses: the flexion angle of the associated joint).Bottom right markers near joints. The amount of STA indicated near the marker centroids is calculated as the distance between the measured and the modelled marker positions. The modelled marker movement is a fraction of the measured bone movement (see Sect. 2.1.1). Although the overall movement on the joints is large, the residual STA is similar to the one on the bones. (Color figure online)

volume element. The coordinates are given in a common coordinate system specified using the MRI scanner.

One flat-hand posture was designated as the reference posture. The transformation of each bone from the reference posture to the other postures was found by the point cloud registration algorithm of Hillenbrand (2008). The algorithm determines a statistical global transformation estimate based on the transformations of a very large number of congruent triangles from both point clouds. The transformation estimate can be expressed as a rotation matrix $\left(R_{\mathrm{b}}\right)_{3 \times 3}$ and a translation vector $\left(\mathbf{t}_{\mathrm{b}}\right)_{3 \times 1}$, or an equivalent transformation matrix $\left(T_{\mathrm{b}}\right)_{4 \times 4}$ :
$T_{\mathrm{b}}=\left(\begin{array}{cc}R_{m} b & \mathbf{t}_{\mathrm{b}} \\ \mathbf{0}_{1 \times 3} & 1\end{array}\right)$

By premultiplying the inverse transformation matrices of the bone poses with the marker positions (in homogeneous coordinates), the relative position of the marker is calculated with respect to the bone on which it sits:

$$
\left(\begin{array}{l}
\mathbf{p}_{\mathrm{m}, \mathrm{rel}} \\
1
\end{array}\right)=T_{\mathrm{b}}^{-1}\left(\begin{array}{l}
\mathbf{p}_{\mathrm{m}} \\
1
\end{array}\right) \text {. }
$$

The movement of a marker with respect to the bone is simply the difference of the relative position and the reference position:

$\mathbf{m}_{\mathrm{m}, k}=\mathbf{p}_{\mathrm{m}, \mathrm{rel}, k}-\mathbf{p}_{\mathrm{m}, \mathrm{ref}}$,

where $\mathbf{m}_{\mathrm{m}, k}$ is the marker movement associated with posture $k, \mathbf{p}_{\mathrm{m}, \mathrm{rel}, k}$ is the relative marker position in posture $k$ and $\mathbf{p}_{\mathrm{m}, \text { ref }}$ is the reference marker position.

To give anatomical meaning to the marker movement, bone coordinate systems (BCS) are introduced for each bone. According to the recommendations of the International Society of Biomechanics (Wu et al. 2005), the $x$-axis points in palmar direction, the $y$-axis in proximal and the $z$-axis in radial direction. The axis can either be placed manually or automated, e.g. using a principal component analysis (see caution remarks on this at the end of Sect. 2.2). The marker movement is transformed to the BCS by left-multiplying with the appropriate transformation matrix:

$$
\left(\begin{array}{c}
{ }^{\mathrm{B}} \mathbf{m}_{\mathrm{m}} \\
1
\end{array}\right)={ }^{\mathrm{B}} T_{\mathrm{MRI}}\left(\begin{array}{l}
\mathbf{m}_{\mathrm{m}} \\
1
\end{array}\right)
$$

where ${ }^{\mathrm{B}} \mathbf{m}_{\mathrm{m}}$ is the marker movement in bone coordinates and $\left({ }^{\mathrm{B}} T_{\mathrm{MRI}}\right)_{4 \times 4}$ is the transformation matrix from the MRI coordinate system to the BCS.

The markers on joints do not belong to any particular one of the two adjacent bones. As a first step, we arbitrarily calculated the relative movement of the markers on joints with respect to the bone proximal to the joint, except for the IMC12 marker, which was associated with the MC1 bone.

Then, the movement was filtered to only include movement in the longitudinal direction of the bone and in the palmar/dorsal direction because the registration of the longitudinal bone orientation-therefore, the measurement of the sideward marker movement—are quite noisy. The filter consists of setting the $z$-component of the motion to zero:

${ }^{\mathrm{B}} \mathbf{m}_{\mathrm{m}, \text { filt }}=\left(\begin{array}{c}{ }^{\mathrm{B}} m_{\mathrm{m}, \mathrm{x}} \\ { }^{\mathrm{B}} m_{\mathrm{m}, \mathrm{y}} \\ 0\end{array}\right)$,

where ${ }^{\mathrm{B}} \mathbf{m}_{\mathrm{m} \text {, filt }}$ is the filtered marker movement and ${ }^{\mathrm{B}} m_{\mathrm{m}, \mathrm{x}}$ and ${ }^{\mathrm{B}} m_{\mathrm{m}, \mathrm{y}}$ are the $x$ - and $y$-components of ${ }^{\mathrm{B}} \mathbf{m}_{\mathrm{m}}$.

The filtered relative marker positions are shown in Fig. 2 (bottom). The marker colour corresponds to the flexion angle 
Table 1 List of hand postures for STA measurements

\begin{tabular}{ll}
\hline Posture number & Posture description \\
\hline 1. & Flat hand with fingers and thumb adducted \\
2. & Relaxed posture \\
3. & Fingers and thumb spread apart and maximally extended \\
4. & Finger closed and maximally extended, thumb maximally extended \\
5. & MCP flexed, PIP and DIP extended \\
6. & Thumb flexed, fingers extended \\
7. & PIP and DIP maximally flexed, MCP extended \\
8. & Flat hand, closed fingers, finger MCP abducted in ulnar direction \\
9. & Flat hand, closed fingers, finger MCP abducted in radial direction \\
10. & Maximal palm arching \\
11. & Holding a cylindrical object as if it was a screw driver \\
12. & Max. extension of thumb MCP and IP \\
13. & Opposition of thumb and little finger pads, maximal flexion of thumb CMC, PIP and DIP extended \\
14. & Thumb tip touches middle finger PIP, thumb MCP maximally flexed \\
15. & Maximal dorsal-ulnar abduction of thumb MCP \\
16. & Thumb tip touches ring and little finger DIP, maximal radial-palmar abduction of thumb MCP \\
17. & Opposition of thumb and index finger tip (pinch grip) \\
18. & Fingernails of thumb and middle finger touch, with the PIP and DIP joints moderately flexed \\
19. & Thumb tip touches ring finger DIP, with MCP of index, middle and ring finger radially abducted \\
20. & Fingers spread apart with MCP flexed \\
\hline
\end{tabular}

of the joint that seems to most influence the marker movement: the DIP joints for the markers on the fingernails, on the DIP joints and on the middle phalanges; the PIP joints for the markers on the PIP joints and on the proximal phalanges; and the MCP joints for the markers on metacarpal bones.

The skin motion is clearly related to the joint angles of the nearby joints. For the purpose of interpolation, we represented the relative motion of the bones in screw axis notation (Waldron and Schmiedeler 2008):

$$
\begin{gathered}
T_{\mathrm{b}, \text { rel }, k}=T_{\mathrm{b}, \text { prox }, k}^{-1} T_{\mathrm{b}, \text { dist }, k}, \\
\left(\begin{array}{lr}
R & \mathbf{t} \\
\mathbf{0}_{1 \times 3} & 1
\end{array}\right)=T_{\mathrm{b}, \text { rel }, k}, \\
\mathbf{u}=\left(\begin{array}{l}
r_{3,2}-r_{2,3} \\
r_{1,3}-r_{3,1} \\
r_{2,1}-r_{1,2}
\end{array}\right), \\
\phi=\operatorname{sign}\left(\mathbf{u}^{\mathrm{T}} \mathbf{t}\right) \operatorname{acos}\left(\frac{1}{2}\left(\sum \operatorname{diag}(R)-1\right)\right), \\
\mathbf{p}_{\text {screw }}=\frac{\mathbf{u}_{\text {screw }}^{\mathrm{T}} \mathbf{t}}{2(1-\cos \phi)}\left(I_{3 \times 3}-R^{\mathrm{T}}\right) \mathbf{t}, \text { and } \\
\mathbf{a}_{\text {screw }}=\frac{1}{2 \sin \phi} \mathbf{u},
\end{gathered}
$$

where $T_{\mathrm{b}, \text { rel }, k}$ is the relative transformation of the distal bone with respect to the proximal bone in posture $k, T_{\mathrm{b} \text {,prox }}^{-1}$ is the inverse of the transformation from the reference pose to pose $k$ of the proximal bone and $T_{\mathrm{b} \text {,dist }}^{-1}$ the same of the distal bone, $R$ and $\mathbf{t}$ are the rotation and translation components of $T_{\mathrm{b}, \text { rel }, k}$, $\mathbf{u}$ is an auxiliary vector, $r_{m, n}$ is the element at the $m$-th row and $n$-th column of $R, \phi$ is the rotation angle, $h_{\text {screw }}$ is the pitch of the screw axis, $\mathbf{p}_{\text {screw }}$ is a point on the screw axis, $I_{3 \times 3}$ is the $3 \times 3$ unit matrix and $\mathbf{a}_{\text {screw }}$ is the orientation of the screw axis.

Screw axis notation divides the transformation into a rotation around an axis in space (defined by $\mathbf{p}_{\text {screw }}, \mathbf{a}_{\text {screw }}$ and $\phi$ ) and a translation along this axis, proportional to the joint angle (defined by $h_{\text {screw }}, \mathbf{a}_{\text {screw }}$ and $\phi$ ). The joint angle is divided by 2 and the motion is transformed back into a transformation matrix:

$$
\begin{gathered}
\phi_{\mathrm{m}}=\frac{\phi}{2}, \\
R_{\mathrm{m}}=\operatorname{rot}\left(\mathbf{a}_{\text {screw }}, \phi_{\mathrm{m}}\right), \\
\mathbf{t}_{\mathrm{m}}=h_{\text {screw }} \phi_{\mathrm{m}} \mathbf{a}_{\text {screw }}, \text { and } \\
T_{\mathrm{m}, k}=\left(\begin{array}{cc}
R_{\mathrm{m}} & \left(I_{3 \times 3}-R_{\mathrm{m}}\right) \mathbf{p}_{\text {screw }}+\mathbf{t}_{\mathrm{m}} \\
\mathbf{0}_{3 \times 1} & 1
\end{array}\right),
\end{gathered}
$$

with

$$
\begin{aligned}
& \operatorname{rot}(\mathbf{a}, q): \\
& =\left(\begin{array}{lll}
c+c^{\prime} a_{x}^{2}, & c^{\prime} a_{x} a_{y}-a_{z} s, & c^{\prime} a_{x} a_{z}+a_{y} s \\
c^{\prime} a_{x} a_{y}+a_{z} s, & c+c^{\prime} a_{y}^{2}, & c^{\prime} a_{y} a_{z}-a_{x} s \\
c^{\prime} a_{x} a_{z}-a_{y} s, & c^{\prime} a_{y} a_{z}+a_{x} s, & c+c^{\prime} a_{z}^{2}
\end{array}\right), \\
& c=\cos q \text {, } \\
& c^{\prime}=1-\cos q \text {, } \\
& s=\sin q, \\
& \mathbf{a}=\left(a_{x}, a_{y}, a_{z}\right)^{\mathrm{T}}, \quad \text { and } \quad\|\mathbf{a}\|=1 \text {, }
\end{aligned}
$$

where $T_{\mathrm{m}, k}$ is the interpolation of $T_{\mathrm{b}, \mathrm{rel}, k}$ at half of the movement. 
The resulting transformation was applied to the reference marker position to calculate model the joint motion-dependent marker position:

$$
\left(\begin{array}{c}
\mathbf{p}_{\mathrm{m}, \bmod , k} \\
1
\end{array}\right)=T_{\mathrm{m}, k}\left(\begin{array}{c}
\mathbf{p}_{\mathrm{m}, \mathrm{ref}} \\
1
\end{array}\right)
$$

where $\mathbf{p}_{\mathrm{m}, \bmod , k}$ is the modelled marker position.

The difference between modelled and measured marker position was formed and transformed and filtered analogously to Eqs. (1) and (2):

$$
\begin{aligned}
& \mathbf{d}_{\mathrm{m}, k}=\mathbf{p}_{\mathrm{m}, \mathrm{mod}, k}-\mathbf{p}_{\mathrm{m}, \mathrm{rel}, k}, \\
& \left(\begin{array}{c}
{ }^{\mathrm{B}} \mathbf{d}_{\mathrm{m}, k} \\
1
\end{array}\right)={ }^{\mathrm{B}} T_{\mathrm{MRI}}\left(\begin{array}{c}
\mathbf{d}_{\mathrm{m}, k} \\
1
\end{array}\right), \\
& { }^{\mathrm{B}} \mathbf{d}_{\mathrm{m}, \mathrm{filt}, k}=\left(\begin{array}{c}
{ }^{\mathrm{B}} d_{\mathrm{m}, \mathrm{x}, k} \\
d_{\mathrm{m}, \mathrm{y}, k} \\
0
\end{array}\right)
\end{aligned}
$$

The STA shown in Fig. 2 (bottom) are the maxima of the 2-norms of the marker movement for the markers on bones and the maxima of the 2-norms of the model-measurement differences for the markers on joints:

$$
\begin{gathered}
\mathrm{STA}_{\text {bone }}=\max _{k}\left(\|\|^{\mathrm{B}} \mathbf{m}_{\mathrm{m}, \text { filt }, k} \|\right) \text { and } \\
\text { STA }_{\text {joint }}=\max _{k}\left(\left\|\left.\right|^{\mathrm{B}} \mathbf{d}_{\mathrm{m}, \text { filt }, k}\right\|\right) .
\end{gathered}
$$

It turned out that this kind of interpolation explains on average about $50 \%$ of the marker motion on joints and typically reduces the maximum movement by as much as $60 \%$.

For compensating skin motion, Zhang et al. (2003) modelled marker movement as a rotation around the joint axis with an angle proportional to the joint angle. Another method is to do a regression fit between the marker motion and the joint angles (Dexmart 2009).

\subsubsection{Number of markers}

There are different approaches concerning the number of markers per segment (MPS). Typically, at least one base segment is fitted with three or more markers, which define its six-dimensional pose (position and orientation). For the other segments, some works use one MPS (Zhang et al. 2003; Miyata et al. 2004; Cerveri et al. 2007; Choi 2008; Metcalf et al. 2008) and some use three MPS (Cerveri et al. 2007; Chang and Pollard 2007a).

With one MPS, there is ample space on the segments, and relatively large markers can be used. Using three MPS, the size of the markers is limited, requiring a high resolution capture system. The total number of markers that need to be labelled and the risk of marker occlusions are higher. However, the relatively stable distances between the markers on a segment can also facilitate automatic labelling. The risk of occlusion of all markers on a segment is actually lower than in the one-MPS setup. Errors due to STAs are mitigated when using three MPS because the severity of the skin movement is different across different positions of the skin.

The identification of axis parameters in setups with one MPS depends on the whole kinematic chain down to the base segment. Consequently, errors in proximal axis parameters also affect more distal joints. In setups with three MPS, each joint can be treated separately.

We have successfully used a three-MPS setup with marker diameters of $3 \mathrm{~mm}$ in a $7 \mathrm{MX} 3+$ camera VICON setup placing the cameras in $75 \mathrm{~cm}$ distance to the observed object in a semicircle setup. With this camera setup, we were able to track small female hands (67 mm small finger length).

Placing more than three markers on the skin may require even smaller markers and correspondingly higher camera resolution. However, markers can be fixed on a segment at certain relative positions using marker trees. This way, four markers can easily be placed, allowing a fairly large set of unique tetrahedrons that can be used to automatically label the segments of the hand (Gierlach et al. 2012). The resulting markers can be made so small that we were able to track hands of a 7-year-old European child.

\subsection{MRI}

MRI as a method for studying joint kinematics was introduced by Hirsch et al. (1996) with a tarsal (foot) joint as an example. The MRI images are segmented slice-by-slice to form the point cloud of the bone. The movement of a bone from one image to the next is determined by registration, i.e. matching the point clouds. The joint parameters, such as positions and orientations of joint axes, are fit to the measured relative motions of one bone with respect to another. The first application of the MRI method on human finger joints was documented by Miyata et al. (2005). A complete MRI-based hand kinematics model, covering the whole range of motion of all joints, including abduction movements, was first presented by van der Smagt and Stillfried (2008). The model was later improved to incorporate non-intersecting joint axes for the thumb (Stillfried and van der Smagt 2010).

For registration, Hirsch et al. (1996) match the principal axes of the point clouds, which are determined by a principal component analysis (PCA). This method seems to work fine in tarsal joints because of the particular shapes of the tarsal bones. But, this is not the case in finger bones. PCA works by determining the eigenvectors $\mathbf{u}_{i}$ and eigenvalues $\lambda_{i}$ of the covariance matrix $S$ of the point cloud (Bishop 2006):

$S \mathbf{u}_{i}=\lambda_{i} \mathbf{u}_{i}$,

with

$S=\frac{1}{N} \sum_{n=1}^{N}\left(\mathbf{x}_{n}-\overline{\mathbf{x}}\right)\left(\mathbf{x}_{n}-\overline{\mathbf{x}}\right)^{\mathrm{T}}$ and 


$$
\overline{\mathbf{x}}=\frac{1}{N} \sum_{n=1}^{N} \mathbf{x}_{n},
$$

such that

$\lambda_{1}>\lambda_{2}>\lambda_{3}$ and

$\mathbf{u}_{i}^{\mathrm{T}} \mathbf{u}_{i}=1$,

and where $\mathbf{x}_{n}$ are the coordinates of the $n$-th point of the point cloud.

The first principal component (PC) is the eigenvector $\mathbf{u}_{1}$ with the largest eigenvalue $\lambda_{1}$, the second PC is the eigenvector $\mathbf{u}_{2}$ with the second largest eigenvalue $\lambda_{2}$ and so on; i.e. the eigenvalues need to be clearly separable in all point cloud representations of the same bone. However, we found that in finger bones, only the first PC is clearly separated-the ranges of eigenvalues for the second and third PC overlap. So, more subtle features of the bones need to be exploited, as, e.g. with the method of Hillenbrand (2008). In that method, a very large number of congruent point triples from both point clouds are drawn and the motions that align the point triples are analysed statistically to find the best motion that aligns the two point clouds.

\subsection{Axis description and parameter identification}

The motion of many joints can be approximated by one or more rotation axes. The parameters of a rotation axis are its position and its orientation. These parameters can be found by minimising suitable cost functions. A simple case is the hinge joint, described by a single rotation axis. If the relative motion of markers is supposed to be modelled by a hinge joint, a circle can be fit to measured marker positions, as, e.g. described by Chang and Pollard (2007b). The normal of the circle plane is parallel to the axis orientation, and the axis position is defined by the centre of the circle. The cost function is the sum of the squared distances between all measured marker positions and their closest points on the circle. This circle-fitting method can also work in 2-DoF joints if the range of motion around the dominant axis is at least twice as large as the range of motion around the secondary axis. Joints with three intersecting, mutually orthogonal rotation axes are also called spherical joints. A rigid object can rotate around a spherical joint into any arbitrary orientation, independent of the orientation of the rotation axes. Therefore, for these joints, only the centre of rotation (intersection point of the three axes) is of interest, and the orientations of the axes can be chosen arbitrarily. The positions of markers on a rigid object rotating around a spherical joint lie on concentric spheres. A closed-form method for finding the centre of such a sphere or set of spheres is presented by Chang and Pollard (2007a), again with the sum of squared distances of the markers from the spheres as the cost function. There are also more complex optimisations which require the identification of the joint angle of each recorded posture. These include optimisations which model the skin movement over the bone (Zhang et al. 2003) and simultaneous optimisations of multiple axes of a kinematic chain.

If bone pose information is available, e.g. from the registration of point clouds from MRI images, the optimisation cost functions can include the rotational error as well as the translational error between the measured and modelled bone poses. In our analysis based on MRI images, we subdivided the optimisation problem to keep the dimension of the search space low. We first optimised the axis orientations and subsequently the axis positions. For the axis orientations, we used a nested optimisation approach: In the outer optimisation, the axis orientations were optimised, and in each iteration of the outer cost function the joint angles were optimised in a set of inner optimisations. The cost functions of the inner optimisations were the rotational distances between the measured and modelled bone orientations of each posture. The measured orientations were defined with respect to a reference pose, while the modelled orientations were calculated by rotating the reference pose around the respective axes. The cost function of the outer optimisation was the sum of the minima of the inner cost functions.

Assume that we have MRI images of a hand in one reference posture and in a number of $n_{\mathrm{p}}$ additional postures. Consider two bones, connected by a joint with $n_{\mathrm{a}}$ rotation axes $\left(n_{\mathrm{a}} \in\{1,2,3\}\right)$. The point clouds of the bones are segmented from the MRI image volumes and shall be denoted $\mathcal{P}_{\text {ref }}$ for the point cloud of the proximal bone in the reference posture, and $\mathcal{P}_{p}$ for the point cloud of the proximal bone in the $p$-th additional posture; $\mathcal{D}_{\text {ref }}$ and $\mathcal{D}_{p}$ for the distal bone. The point clouds are given in the coordinate system of the MRI machine.

The output of the registration algorithm shall be given by a rotation matrix $R_{p \text {,prox }}$ and a translation vector $\mathbf{t}_{p \text {,prox }}$ which rotate and translate $\mathcal{P}_{\text {ref }}$ so that it matches $\mathcal{P}_{p}$, and by $R_{p \text {, dist }}$ and $\mathbf{t}_{p \text {, dist }}$ that rotate and translate $\mathcal{D}_{\text {ref }}$ to match $\mathcal{D}_{p}$. For the sake of modelling the joint, consider the proximal bone to be fixed and the distal bone to move relative to the proximal bone. The relative pose of the distal bone with respect to the proximal bone shall be described by a rotation matrix $R_{p}$ and translation vector $\mathbf{t}_{p}$ :

$$
\begin{gathered}
R_{p}=R_{p, \text { prox }}^{-1} R_{p, \text { dist }} \text { and } \\
\mathbf{t}_{p}=R_{p, \text { prox }}^{-1}\left(\mathbf{t}_{p, \text { dist }}-\mathbf{t}_{p, \text { prox }}\right) .
\end{gathered}
$$

The orientations of the rotation axes are identified in the outer optimisation by minimising the mean angular distance between the measured and modelled relative orientations of the bone: 


$$
\begin{aligned}
& \left\{\mathbf{a}_{1, \text { opt }}, \ldots, \mathbf{a}_{n_{\mathrm{a}}, \text { opt }}\right\}=\underset{\left\{\mathbf{a}_{1}, \ldots, \mathbf{a}_{n_{\mathrm{a}}}\right\}}{\arg \min } \sum_{p=1}^{n_{\mathrm{p}}} \\
& \text { angle }\left(R_{p} R_{p, \text { mod }}^{-1}\left(\mathbf{a}_{1}, \ldots, \mathbf{a}_{n_{\mathrm{a}}}, q_{1, p, \mathrm{opt}}, \ldots, q_{n_{\mathrm{a}}, p, \mathrm{opt}}\right)\right),
\end{aligned}
$$

where $\mathbf{a}_{1}, \ldots, \mathbf{a}_{n_{\mathrm{a}}}$ are the orientation vectors of the rotation axes, $q_{1, p, \mathrm{opt}}, \ldots, q_{n_{\mathrm{a}}, p \text {,opt }}$ are the optimal joint angles found by the inner optimisation and $R_{p \text {,mod }}$ is the modelled relative orientation of the bone in posture $p$. The angle of a rotation described by a rotation matrix $R$ is calculated as follows:

$\operatorname{angle}(R)=\arccos \left(\frac{\operatorname{trace}(R)-1}{2}\right)$.

The angular distance, angle $\left(R_{1} R_{2}^{-1}\right)$, between two orientations $R_{1}$ and $R_{2}$ can be regarded as analogue to the Euclidean distance $\left\|\mathbf{x}_{1}-\mathbf{x}_{2}\right\|$ between two positions $x_{1}$ and $x_{2}$. The modelled orientation of the bone results from rotating the bone in its reference posture around the rotation axes of the joint, which corresponds to a product of rotation matrices:

$$
\begin{array}{r}
R_{p, \bmod }\left(\mathbf{a}_{1}, \ldots, \mathbf{a}_{n_{\mathrm{a}}}, q_{1, p}, \ldots, q_{n_{\mathrm{a}}, p}\right)= \\
\operatorname{rot}\left(\mathbf{a}_{1}, q_{1, p}\right) \cdots \operatorname{rot}\left(\mathbf{a}_{n_{\mathrm{a}}}, q_{n_{\mathrm{a}}, p}\right) \\
=: \prod_{a=1}^{n_{\mathrm{a}}} \operatorname{rot}\left(\mathbf{a}_{a}, q_{a, p}\right),
\end{array}
$$

where $q_{a, p}$ is the rotation angle around axis $a$ in posture $p$.

The inner optimisation returns, for given axis orientations, the joint angles that minimise the angular distance between the measured and modelled bone orientations:

$$
\begin{aligned}
& \left\{q_{1, p, \mathrm{opt}}, \ldots, q_{n_{\mathrm{a}}, p, \mathrm{opt}}\right\}=\underset{\left\{q_{1, p}, \ldots, q_{n_{\mathrm{a}}, p}\right\}}{\arg \min } \\
& \quad \operatorname{angle}\left(R_{p} R_{p, \text { mod }}^{-1}\left(\mathbf{a}_{1}, \ldots, \mathbf{a}_{n_{\mathrm{a}}}, q_{1, p}, \ldots, q_{n_{\mathrm{a}}, p}\right)\right) .
\end{aligned}
$$

The positions of the rotation axes are identified by minimising the mean Euclidean distance between the measured and modelled position of the bone centroid:

$$
\begin{aligned}
& \left\{\mathbf{p}_{1, \text { opt }}, \ldots, \mathbf{p}_{n_{\mathrm{a}}, \text { opt }}\right\}= \\
& \underset{\left\{\mathbf{p}_{1}, \ldots, \mathbf{p}_{n_{\mathrm{a}}}\right\}}{\arg \min } \sum_{p=1}^{n_{\mathrm{p}}}\left\|\mathbf{c}_{p}-\mathbf{c}_{p, \bmod }\left(\mathbf{p}_{1}, \ldots, \mathbf{p}_{n_{\mathrm{a}}}\right)\right\|,
\end{aligned}
$$

where $\mathbf{p}_{a}$ is a point on axis $a, \mathbf{c}_{p}$ is the measured centroid position and $\mathbf{c}_{p \text {, mod }}$ is the modelled centroid position. The bone centroid $\mathbf{c}_{\text {ref }}$ in the reference posture is calculated as the mean of all points $\mathbf{x}$ in the point cloud of the bone:

$\mathbf{c}_{\mathrm{ref}}=\frac{1}{n_{\mathrm{x}}} \sum_{i=1}^{n_{\mathrm{x}}} \mathbf{x}_{i}$,

where $n_{\mathrm{x}}$ is the number of points in $\mathcal{D}_{\text {ref }}$. The position of the bone centroid in the other measured postures is calculated by transforming the reference centroid by the measured relative motion:

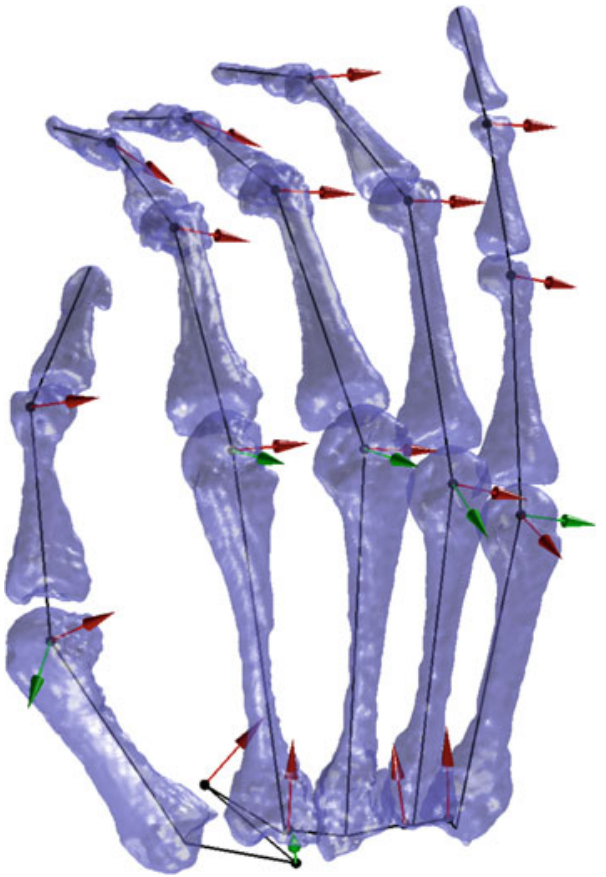

Fig. 3 A kinematic hand model based on the objective functions in Sect. 2.3, in two-DoF joints the second axis is in green colour. From Stillfried et al. (2012). (Color figure online)

$\mathbf{c}_{p}=R_{p} \mathbf{c}_{\mathrm{ref}}+\mathbf{t}_{p}$.

The modelled position of the bone centroid is calculated using the optimal axis orientations and joint angles, $\left\{\mathbf{a}_{1, \mathrm{opt}}, \ldots, \mathbf{a}_{n_{\mathrm{a}}, \mathrm{opt}}\right\}$ and $\left\{q_{1, p, \mathrm{opt}}, \ldots, q_{n_{\mathrm{a}}, p, \mathrm{opt}}\right\}$, and the to-be-optimised axis positions $\left\{\mathbf{p}_{1}, \ldots, \mathbf{p}_{n_{\mathrm{a}}}\right\}$ :

$$
\begin{aligned}
& \mathbf{c}_{p, \bmod }\left(\mathbf{p}_{1}, \ldots, \mathbf{p}_{n_{\mathrm{a}}}\right)= \\
& \prod_{a=1}^{n_{\mathrm{a}}}\left(\operatorname{rot}\left(\mathbf{a}_{a, \mathrm{opt}}, q_{a, p, \mathrm{opt}}\right)\right) \mathbf{c}_{\mathrm{ref}}+ \\
& \sum_{a=1}^{n_{\mathrm{a}}}\left(\prod_{b=1}^{a-1}\left(\operatorname{rot}\left(\mathbf{a}_{b, \mathrm{opt}}, q_{b, p, \mathrm{opt}}\right)\right) \mathbf{p}_{a}\right. \\
&\left.\quad-\prod_{b=1}^{a}\left(\operatorname{rot}\left(\mathbf{a}_{b, \mathrm{opt}}, q_{b, p, \mathrm{opt}}\right)\right) \mathbf{p}_{a}\right) .
\end{aligned}
$$

In the case of intersecting axes, Eq. (4) simplifies to:

$$
\mathbf{c}_{p, \bmod }(\mathbf{p})=\prod_{a=1}^{n_{\mathrm{a}}}\left(\operatorname{rot}\left(\mathbf{a}_{a, \mathrm{opt}}, q_{a, p, \mathrm{opt}}\right)\right)\left(\mathbf{c}_{\mathrm{ref}}-\mathbf{p}\right)+\mathbf{p} .
$$

A kinematic hand model based on above equations is shown in Fig. 3. 


\subsection{Instantaneous axis of rotation}

When studying joint motion, it is useful to consider the concept of the instantaneous axis of rotation. The movement between any two poses of a rigid body can be described as a rotation around an axis in space and a translation along that axis. The axis is called helical axis, and in the case of infinitesimal movements, instantaneous helical axis (Woltring et al. 1987). If there is no translation along the axis, or if the translation is neglected, the axis is called instantaneous axis of rotation (IAR).

In ideal hinge joints, the IAR stays at the same location throughout the whole movement. An example of an 1-DoF joint with a varying IAR is a four-bar linkage. Also, in biological joints, the IAR changes position during movement. This occurs because the curvature of the joint surface is not exactly the same in all parts of the surface, and the motion is additionally guided by ligaments.

For example, the curvature for a finger PIP joint is larger when the finger is close to a stretched-out position and smaller when it is bent (van Nierop et al. 2008). This is significant in the modelling of finger dynamics, as the moment arms of the tendons are calculated as the perpendicular distance from the IAR. Just to mention another approach, a different method avoiding the calculation of the IAR for moment arms could be employed if data about the muscle-tendon excursion is available. In that case, the tendon moment arms could be obtained by the derivative of the muscle-tendon excursion with respect to joint angle.

Still, these joints are often approximated as simple hinge joints, due to several reasons. Firstly, a moving IAR is more complex than a fixed one, requiring additional parameters to describe the motion. Secondly, the IAR cannot be measured exactly, as infinitesimal movements are impossible to measure. Thirdly, even approximating the IAR is difficult because this requires the measurement of small movements, leading to a low ratio of measured movement to measurement error (signal-to-noise ratio). The last problem can be overcome by smoothing and interpolating the motion with splines, as described by Woltring et al. (1987).

\subsection{Synergies}

As mentioned in the introduction, synergies reduce the complexity of common hand postures by finding couplings of joint angles. These couplings can be mechanical, as for example the coupling between the PIP and DIP joint. But, they can also be a constraint by the control system (central and peripheral nervous system) on the hand postures: even though the hand is capable of many more postures, in most situations only a subset of the postures is used. A simple way of finding the postural synergies within a set of measured postures is described by Santello et al. (1998): the joint angles of the measured postures are regarded as points in a space whose dimension is the number $n_{\mathrm{a}}$ of joint axes in the hand. On this set of points, a PCA is performed (see Eq. (3), here with $\left.i \in\left\{1, \ldots, n_{\mathrm{a}}\right\}\right)$. The sum of eigenvalues of the first $n$ PC divided by the sum of eigenvalues of all $\mathrm{PC}$ is the variance $v$ of the joint angles that is explained by these $n$ PC (Bishop 2006):

$v=\sum_{i=1}^{n} \lambda_{i} / \sum_{i=1}^{n_{\mathrm{a}}} \lambda_{i}$.

Santello (Santello et al. 1998) shows that many grasp shapes can be achieved by controlling two or three synergies only: the grasps of 57 imagined objects could be reproduced by two synergies with an average $v$ of $84 \%$ and by three synergies with an average $v$ of $90 \%$.

The concept of synergies can be extended to forces (Gabiccini et al. 2011). A related topic is complexity reduction in sensing, e.g. processing the signals from some hundred mechanoreceptors in the fingertips into the perception of the shape of an object.

\section{Musculoskeletal models}

The musculoskeletal anatomy of the hand and wrist is one of the most complex system of the human body. It consists of 27 bones and 45 muscles with at least 23 degrees of freedom at the joints, if the wrist is considered as a 2-DoF joint. The motions of the fingers are controlled by a combination of extrinsic muscles: flexor digitorum profundus (FDP), flexor digitorum superficialis (FDS), extensor digitorum (ED) and the intrinsic muscles: dorsal and palmar interossei and lumbricals (4).

A pioneer in describing human fingers using a musculoskeletal model was Landsmeer (1961). In his paper, the 2-D finger movement patterns are described depending on the combination of the agonistic and antagonistic muscles activation mechanisms.

There are many challenges in modelling the musculoskeletal anatomy of the hand: routing of the long poly-articular muscles via the joints; modelling the extensor web of the fingers (Fig. 4); implementing the inter-tendon and intermuscle connections and joint coupling of the fingers and wrist. These topics are addressed in several existing hand and finger models.

\subsection{Intrinsic finger muscles and the extensor web}

The interossei and lumbricals form the intrinsic muscles which originate in the palm of the hand and inserting into the extensor web of the fingers or thumb (Fig. 4). The lumbricalis originates from the FDP tendon and inserts into the 


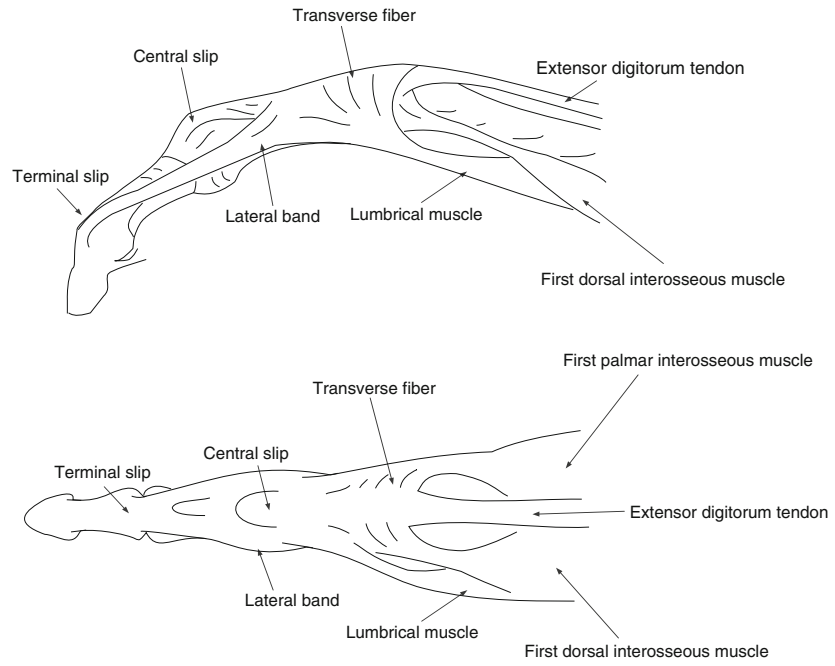

Fig. 4 Extensor web of the index finger of a right hand including extensor digitorum tendon, lumbrical and interossei muscles: top lateral view; bottom dorsal view

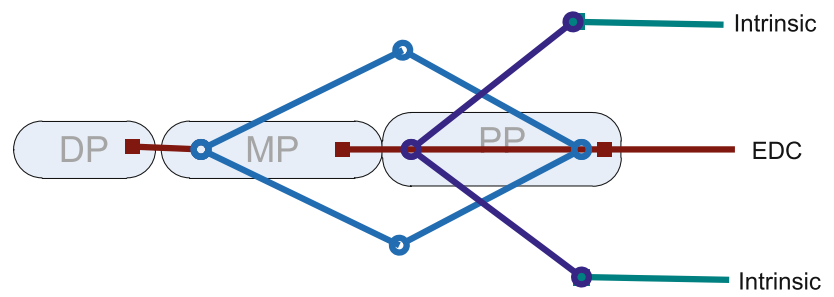

Fig. 5 Delft Hand Model structure of the extensor web with the EDC and intrinsic muscles included

extensor mechanism of the fingers, making it the only muscle in the human body not directly being connected to the bones. Owing to its tendon-based origin, it can affect the FDP flexion force, as well as the extension of the distal finger joints. Furthermore, the lumbricalis is thought to be an MCP joint flexor and PIP and DIP joint extensor.

Although the extrinsic flexors are expected to be the main force-producing muscles in power grip due to its inherent stability, previous studies observed high activation levels of the intrinsic muscles in this grip (Long et al. 1970; SanchoBru et al. 2001). Moreover, Kozin et al. (1999) observed a $49 \%$ reduction of power grip force when the intrinsic muscles were paralysed by a nerve block. This effect was even stronger on pinch grip with a $85 \%$ pinch force reduction due to the nerve block. To increase the understanding of the role of the intrinsic muscles of the hand, musculoskeletal models can be of great use.

The first 3-D normative hand model which includes the intrinsic hand musculature was introduced by An et al. (1979). This model consists of four independent fingers. For each of the fingers, the full muscular anatomy was implemented, including the extrinsic tendons and the intrinsic hand muscles. The model was based on the anatomy of 10 normal hand specimens. The anatomical data were derived using ten- don markers in combination with 2-D X-rays. A two-point technique was implemented to model the lateral bands of the extensor mechanism at the PIP joint resulting in jointdependent moment arms at the PIP joints. This model was later implemented in the commercial software ANYBODY for the thumb (Wu et al. 2009) and the index finger (Wu et al. 2010).

The extensor mechanism of the fingers, including the intrinsic musculature, has been modelled extensively by Valero-Cuevas et al. (1998) and Sancho-Bru et al. (2001). The model of the extensor mechanism was based on the Winslow's rhombus representation (Zancolli 1979).

Most extensor models described here give a complete representation of the intrinsic musculature and the extensor web at the level of the individual fingers. However, the coupling between the fingers and interaction with the wrist was not implemented in the model. This is a limitation when investigating human grasping.

\subsection{Wrist-finger joint coupling}

The interaction between fingers and the wrist is an important aspect in grasping. During grasping, the grip force depends on the wrist orientation; when the wrist is flexed, the flexion force of the finger is reduced due to slackening of the extrinsic finger flexors (Dempsey and Ayoub 1994). Therefore, the wrist needs to be stabilised in a slightly extended position to achieve maximum grasp force ( $\mathrm{Li}$ et al. 2002). In addition, during finger flexion, the extrinsic tendons exert a flexion moment at the wrist which will need to be compensated for by the wrist extensors (Snijders et al. 1987).

Holzbaur et al. (2005) developed an upper extremity model including the shoulder and elbow, the wrist, thumb and index finger. This model can provide some insight into the wrist-finger joint coupling. However, since it does not include the intrinsic hand muscles, it can therefore not be used to fully evaluate hand function. In addition to the model, they presented an extensive study of upper limb musculature in ten living subjects using MRI (Holzbaur et al. 2006, 2007). This study provides valuable data on the wrist and extrinsic hand muscle anatomy.

\subsection{Complete hand and wrist model}

The authors are not aware of a complete musculoskeletal model of the hand and wrist. Such a model incorporates the complete muscle-tendon structures, intrinsic and extrinsic of the forearm and hand, accurate joint axis definitions and segment inertia and can be driven inverse and forward dynamic. Therefore, a complete biomechanical hand and wrist model is being developed at Delft University of Technology. The model comprises the lower arm, wrist, hand, thumb and four fingers. The model is Fortran based and developed on 
the same platform as the Delft Shoulder and Elbow Model (van der Helm 1994).

The Delft Hand Model consist of 22 rigid bodies: Ulna, radius, the carpal bones as a single body, metacarpals and the phalanges, and it has $23 \mathrm{DoF}$ at the joints. The anatomical structure of the model is based the anatomical data obtained through dissection and 3-D digitisation of the anatomy of one cadaveric lower arm specimen. A total of 45 muscles are implemented. The model of the extensor web of the fingers, including the interossei and lumbricals is depicted in Fig. 5. The inverse-dynamic version of the model uses external forces and joint kinematics as an input, and derives the muscle activation through a minimal-energy optimisation criterion.

Inverse dynamic simulations using the model were performed for MCP flexion motion of the fingers and a pinch grip. In MCP flexion both FDP and FDS were active as well as the lumbricals, while there was no ED activation at the fingers. This indicates that the lumbricals are important in modifying the flexion moment at the MCP joint. For the pinch grip, in addition to the thumb and index finger flexors, the interosseus muscle was active. At the level of the wrist, the wrist extensor muscles showed a substantial activation during pinch grip, showing the importance of including the wrist in a hand model when studying human grasping. In the near future the Delft Hand Model will be validated more extensively to make it suitable for studying a broad range of grasps.

\section{Forward dynamic models}

Dynamic models describe the movement of objects as caused by internal or external forces working on them. For human hand modelling, this means motions as caused by muscle force patterns which results in a tendon/bone movement pattern.

In historical studies before 1867, finger movement was observed visually and by feeling muscles with the hand in healthy and non healthy persons. With the aid of clinical studies hand function was investigated. Later methods used electro stimuli to activate a special region of muscle/s and the effect on the hand was observed. Nevertheless, using these methods not all effects could be explained. In 1867 Duchenne (de Boulogne) helped himself by studying human hand movement using an artificially driven human skeleton. He dissected a human forearm with a hand and replaced the tendon structure by strings which he then 'played' by hand. He noted that a fairly complex 'play' resulted in a 'natural' finger movement.

More than a 100 years later, the facilities for doing such investigations have changed considerably. Cooling systems and precise sensors are available. An et al. carried out basic studies on cadavers investigating tendon excursion and moment arm of index finger muscles by applying a $5 \mathrm{~N}$ weight to the tendons in (An et al. 1983). The joints of the index finger were fixated except one, on which the angle was measured using an electric goniometer. The finger was moved passively and slowly while the displacement of each tendon was measured. Buford et al. (2005) developed these types of measurement to investigate muscle contributions to movement of the MCP. Measuring angle displacement and tendon excursions in a first experiment, muscle moment arms and joint moments were calculated. In a second experiment, he validated the first one by attaching weights to the tendons and measuring moments on the fingertip.

Another important development was the invention of motion tracking systems, from which optical tracking is described in Sect. 2.1. They provided a precise measurement of movement. Regarding the hand, finger movements could be recorded in free movements and couplings between joints could be quantised (Hahn et al. 1995). Similarly, (Leijnse 1998) developed an apparatus which uses anatomical limitations to measure in contact in vivo deep flexor forces and couplings between these.

In the past 10 years, the developments of in vivo measurements have expanded. Now, it is possible to measure tendon forces quasi directly by taking advantage of surgical procedures such as open carpal tunnel release (Dennerlein 2005). But, these occasions are very rare and additional risk and sensory effects have to be taken into account. For example, the use of buckle transducers leads to a change of tendon length, which affects the length-tension curve of the muscle (Fleming and Beynnon 2004). Therefore, additional in situ calibrations are required.

In Pittsburgh, another method was defined after 2000. In order to be able to study the human hand function, without the needs of cadavers, an Anatomically Correct Testbed (ACT) was designed (Chang and Matsuoka 2006; Wilkinson et al. 2003). The test bed tries to mimic parts of the human hand apparatus as close as possible in an attempt at replicating the biomechanical function of the hand.

\subsection{Dynamics and contact tasks}

In a highly complex system like the human hand, many effects play a part in dynamic movement. Moving a human hand raises issues like

1. Joint friction (Alexander 1992),

2. Joint behaviour under different joint loads,

3. Influence of skin and to the tendon attached tissue,

4. Tendon path changes,

and so forth. Also, force transfer is crucial for the human hand as it is made for contact and manipulating purpose. The force output on the finger tip is highly joint dependent (Sect. 3.2). Forces in the human hand, especially in the 
fingers, provide stable grasp and precise manipulation of objects. This includes slipping of the object as well as rotating the object wherever not wanted. For very delicate objects, even deformation has to be avoided. A contrasting function would be the application of a force to deform an object being sculpted.

We focus more on muscle activation patterns and resultant positions/forces. These patterns are a function of the joints moved by the tendons and fingertip force (Dennerlein 2005). Valero-Cuevas et al. (1998) observed that in case of large index fingertip forces the pattern of muscle activation is subject independent. This is a surprising result considering the structural variability in human hands (Schmitt2003).

\subsection{The purpose of the models}

According to An et al. (1983), a major benefit of hand research is the proper reconstruction of the damaged hand. Valero-Cuevas provides a tool for measuring and quantifying anatomical structures which are crucial for grasp abilities of patients who have to undergo surgery (Valero-Cuevas and Laboratory 2000). Kamper is investigating human hands of healthy subjects and stroke patients for rehabilitation (Seo et al. 2010). The ACT hand (Despande 2013) targets various issues, serving as a realistic model for surgeons to drypractice reconstructive surgery, but also to investigate neural control as well as for high-precision teleoperation and prosthetics.

But, there are also different reasons for hand modelling: Leijnse (2012, Personal communication) desires to model effects from tendon sheets and tendon coupling in musician hands with the final goal to explain dystonia and to develop new treatments.

We like to add one more reason studying human hand function. Our incentive is to understand human grasping, to extract functional properties with the final goal to optimise robotic grasping by building mechatronically simpler hands with at least similar grasping and manipulation capabilities. For instance, a robotic system currently under development (see Sect. 1) has an independent joint architecture in the hand, which may or may not be advantageous in grasping. Which independence of joints do we need in 'normal' grasping tasks? Conversely, the control of such an independent architecture is very challenging. By adding synergies (see Sect. 2.5), we can reduce the complexity of control, but we also want to keep a certain, currently unquantified, level of dexterity.

\subsection{The neuroscience of grasping}

Certainly, as described above, there are various per-individual synergies given by interconnections between finger tendons and shared ligaments, which constrain the move- ment of the fingers. Indeed, there is quite some variability between hands, resulting in minute, but sometimes relevant differences between hand use and manipulability since they lead to different synergistic effects and manipulation 'restrictions'. A sufficient description of spinal, cerebellar and cortical causes of synergies is not available. Nevertheless, given the structural components of the neuronal circuitry, there can be little doubt that the brain really works in terms of synergies also when it comes to hand control. The neuronal connections from the motor areas of the neocortex down to the spinal cord are characterised by widely divergent terminations, targeting multiple motorneuron pools (each motorneuron pool innervating a single muscle) (Shinoda et al. 1986, 1986). In addition, the main effect of the so called corticospinal tract is not exerted directly on the motorneurons innervating the muscle, but rather indirectly via a large pool of premotor spinal interneurons (Jankowska 1992; Bortoff and Strick 1993; Isa et al. 2006; Alstermark et al. 2007). Also, spinal premotor neurons have a divergent innervation to target multiple motorneuron pools (Alstermark et al. 1991; Jankowska 1992; Takei and Seki 2010). In other words, all connections, which serve as the infrastructure for neural motor control is naturally synergistic, i.e. they do not target single muscles in a point-to-point fashion. Furthermore, the spinal interneurons feature a rich sensory feedback from skin tactile sensors, muscle tendon organs and muscle spindles (Jankowska 1992). Since spinal premotor interneurons have either excitatory or inhibitory effects on motorneurons, and the two types exists in about equal proportions, sensory feedback evoked during a movement will become an integral part of the motor command issued from the cortex. This circuitry could be used to ensure stability in the interaction between a finger and an object, for example in a grasp, to regulate finger stiffness and additional features (Raphael et al. 2010) including such that remains yet to be discovered.

\section{Outlook}

With the combination of the kinematic hand model, musculoskeletal model and dynamic model, many insights can be obtained which will be applicable in the field of robotic hand design and the clinic.

\subsection{Universal kinematic hand model}

Going back to synergies (Sect. 2.5), we like to take the concept of synergies to a meta-level: while postural synergies reduce the complexity of hand postures and movements within an individual hand, the new 'meta-synergies' would be a small set of parameters that describes the variability of hand kinematics between individuals. For this, we acquire the joint axis parameters of many subjects. With these 'meta- 
synergies', the similarities and differences between subjects can be found. The similarities will form the basis for a general hand model, while the differences will be described with as little parameters as possible. Ideally, with these parameters, the general hand model can be adjusted to fit the kinematics of any individual hand. If the similarities found between human hands will be large compared to the differences, it will be much easier to create kinematic models of individual hands.

With a better kinematic model, the joint angles can be measured more precisely, either with an optical tracking system or a data glove. The accurate joint angles derived using the kinematic model can be of great use as an input for the Delft Hand model.

\subsection{Musculoskeletal hand model}

The proposed Delft hand and wrist model will be the first complete hand and wrist model including the intrinsic musculature and wrist finger coupling. Such a detailed model can provide valuable insights in the underlying mechanisms of hand function.

In addition to the joint kinematics, insights can be obtained in joint forces and the muscle activation patterns using the musculoskeletal model. These activation patterns can serve as input to dynamic measurements (Sect. 4.1). Using preknowledge reduces the area of search for valid muscle activation patterns for a specific movements.

\subsection{Telemanipulation}

An individualised kinematic model of the hand will prove useful, for example, in telemanipulation scenarios, in which a robot hand is used by different operators. A good kinematic model of the operator's hand will improve the mapping between the motions of the operator and the robot.

\subsection{Prosthetics}

Another application of kinematic hand models is the mechanical design of anthropomorphic robot hands. One could for example imagine a modular prosthetic hand the segments of which are adapted to the dimensions of the intact hand in unilateral amputees, or to other body dimensions in bilateral amputees. The rapid prototyping technology used for the iLimb hand (Touch Bionics, Inc., Livingston, UK) precisely enables such modularity.

\subsection{Control}

Improved models of postural synergies (Sect. 2.5) can help to make the control of robot hands much easier. Instead of having to control all joint axes, only a small number of combinations of joint axes is controlled. If the synergies can be implemented in the mechanics of a hand of a robot, that robot will need fewer motors, possibly reducing the weight of either the hand or the forearm, wherever the place of the hand driving elements is foreseen in the design of the robot. Both of these issues are also highly relevant for hand prostheses, where strict constraints on the weight must be taken into account.

\subsection{Clinical diagnostics, training and surgery}

In the clinic, a more detailed knowledge about hand kinematics and dynamics can be used to improve diagnostic and reconstructive surgery. With a computable model, adaptable to every person, first the disease can be diagnosed and different solutions to restore hand functionality can be validated using such models. This applies to functional recovery of hand kinematics as well as the reconstruction of grasp force.

The Delft Hand Model can provide further insights in the mechanical causes of diseases affecting the hand such as osteoarthritis. The model can also be a valuable training tool for future hand surgeons. At a later stage, patient-specific versions of the Delft hand and wrist model can be implemented in the clinical planning by predicting outcomes of surgical procedures such as tendon transfers.

\subsection{Human-robot interaction}

Especially for the case of human robot interaction-for example in household applications - the robot hand can be designed with a more 'natural' behaviour. One major challenge for robotic systems is to deal with 'our' surroundings. Similar kinematics and dynamics are a simple, but effective way to optimise integrability of such robotic systems. Another important advantage is the more intuitive interaction with such devices. Musculoskeletal hand and wrist models can serve as an inspiration for robotic hands, by providing insight in the natural impedance of the musculoskeletal system.

Acknowledgments The authors would like to thank Dr. Marcus Settles from Rechts der Isar hospital, Munich, for the acquisition of the MRI images; Karolina Stonawska and Tobias Lüddemann for the segmentation of the bones and skin markers from the MRI images. This work has been partly sponsored by the EC project STIFF under grant no. 231576, and the EC project The Hand Embodied under grant no. 248587 .

Open Access This article is distributed under the terms of the Creative Commons Attribution License which permits any use, distribution, and reproduction in any medium, provided the original author(s) and the source are credited. 


\section{References}

Alexander R (1992) The human machine. How the body works. Columbia University Press, New York

Alstermark B, Isa T, Pettersson LG, Sasaki S (2007) The C3-C4 propriospinal system in the cat and monkey: a spinal pre-motoneuronal centre for voluntary motor control. Acta Physiol 189(2):123-140

Alstermark B, Isa T, Tantisira B (1991) Integration in descending motor pathways controlling the forelimb in the cat. 18. Morphology, axonal projection and termination of collaterals from $\mathrm{C} 3-\mathrm{C} 4$ propriospinal neurones in the segment of origin. Exp Brain Res 84(3):561-568

An K, Chao E, Cooney W, Linscheid R et al (1979) Normative model of human hand for biomechanical analysis. J Biomech 12(10): 775-788

An K, Ueba Y, Chao E, Cooney W et al (1983) Tendon excursion and moment arm of index finger muscles. J Biomech 16(6):419-425

Bishop CM (2006) Pattern recognition and machine learning. Springer, Heidelberg

Bortoff GA, Strick PL (1993) Corticospinal terminations in two newworld primates: further evidence that corticomotoneuronal connections provide part of the neural substrate for manual dexterity. J Neurosci 13(12):5105-5118

Buford WL, Koh S, Andersen CR, Viegas SF (2005) Analysis of intrinsic-extrinsic muscle function through interactive 3-dimensional kinematic simulation and cadaver studies. J Hand Surg 30(6):1267-1275

Cerveri P, De Momi E, Lopomo N, Baud-Bovy G et al (2007) Finger kinematic modeling and real-time motion estimation. Ann Biomed Eng 35:1989-2002

Cerveri P, Lopomo N, Pedotti A, Ferrigno G (2005) Derivation of centers of rotation for wrist and fingers in a hand kinematic model: methods and reliability results. Ann Biomed Eng 33: 402-412

Chang LY, Matsuoka Y (2006) A kinematic thumb model for the ACT hand. In: Proceedings of the IEEE international conference on robotics and automation (ICRA '06), Orlando, pp 1000-1005

Chang LY, Pollard NS (2007) Constrained least-squares optimization for robust estimation of center of rotation. J Biomech 40(6): $1392-1400$

Chang LY, Pollard NS (2007) Robust estimation of dominant axis of rotation. J Biomech 40(12):2707-2715

Chao E, Opgrande J, Axmear F (1976) Three-dimensional force analysis of finger joints in selected isometric hand functions. J Biomech 9(6):387-396

Choi J (2008) Developing a 3-dimensional kinematic model of the hand for ergonomic analyses of hand posture, hand space envelope, and tendon excursion. Ph.D. thesis, University of Michigan

Dempsey MF, Condon B, Hadley DM (2002) MRI safety review. Seminars in Ultrasound, CT, and MRI 23:392-401

Dempsey PG, Ayoub MM (1994) An investigation of variables influencing sustained pinch strength and evaluation of inter-study variation in independent variable effects. In: Advances in industrial ergonomics and safety VI. Taylor and Francis, London, pp 601-608

Dennerlein JT (2005) Finger flexor tendon forces are a complex function of finger joint movement and fingertip forces. J Hand Therapy 18:120-127

Deshpande AD, Xu Z, Weghe M, Brown BH et al (2013) Mechanisms of the anatomically correct testbed hand. IEEE/ASME Trans Mechatron 18(1):238-250

Dexmart (2009) Deliverable D1.1 kinematic model of the human hand. Tech. rep., Dexmart

Duchenne (de Boulogne) G (1867) Physiologie des mouvements démontrée à l'aide de l'expérimentation éléctrique et de l'observation clinique et applicable à l'étude des paralysies et des défor- mations. J.-B. Baillière et Fils Libraires de l'Académie Impériale de Médecine

Fleming B, Beynnon B (2004) In vivo measurement of ligament/tendon strains and forces: a review. Ann Biomed Eng 32:318-328

Gabiccini M, Bicchi A, Prattichizzo D, Malvezzi M (2011) On the role of hand synergies in the optimal choice of grasping forces. Auton Robots [special issue on RSS2010] 31:235-252

Gierlach D, Gustus A, van der Smagt P (2012) Generating marker stars for $6 \mathrm{~d}$ optical tracking. In: The Fourth IEEE RAS/EMBS international conference on biomedical robotics and biomechatronics, Rome, Italy

Grebenstein M, Albu-Schäffer A, Bahls T, Chalon M, et al. (2011) The DLR hand arm system. In: 2011 IEEE international conference on robotics and automation, Shanghai

Grebenstein M, van der Smagt P (2008) Antagonism for a highly anthropomorphic hand-arm system. Adv Robot 22(1):39-55

Hahn P, Krimmer H, Hradetzky A, Lanz U (1995) Quantitative analysis of the linkage between the interphalangeal joints of the index finger: An in vivo study. J Hand Surg 20(5):696-699

Hillenbrand U (2008) Pose clustering from stereo data. In: Proceedings VISAPP international workshop on robotic perception, Funchal, pp 23-32

Hirsch BE, Udupa JK, Samarasekera S (1996) New method of studying joint kinematics from three-dimensional reconstructions of MRI data. J Am Podiatr Med Assoc 86:4-15

Hollister A, Buford WL, Myers LM, Giurintano DJ et al (1992) The axes of rotation of the thumb carpometacarpal joint. J Orthop Res 10:454-460

Holzbaur K, Delp S, Murray W (2006) Moment-generating capacity of upper limb muscles. J Biomech 39(Supplement 1):S85

Holzbaur KR, Murray WM, Gold GE, Delp SL (2007) Upper limb muscle volumes in adult subjects. J Biomech 40(4):742-749

Holzbaur KRS, Murray WM, Delp SL (2005) A model of the upper extremity for simulating musculoskeletal surgery and analyzing neuromuscular control. Ann Biomed Eng 33:829-840

Isa T, Ohki Y, Seki K, Alstermark B (2006) Properties of propriospinal neurons in the $\mathrm{C} 3-\mathrm{C} 4$ segments mediating disynaptic pyramidal excitation to forelimb motoneurons in the macaque monkey. J Neurophysiol 95(6):3674-3685

Jacobsen S, Iversen E, Knutti D, Johnson R, et al. (1986) Design of the Utah/M.I.T. dextrous hand. In: Proceedings of 1986 IEEE international conference on robotics and automation, vol 3, San Francisco, pp 1520-1532

Jankowska E (1992) Interneuronal relay in spinal pathways from proprioceptors. Prog Neurobiol 38(4):335-378

Kozin SH, Porter S, Clark P, Thoder JJ (1999) The contribution of the intrinsic muscles to grip and pinch strength. J Hand Surg 24(1): 64-72

Kragten GA, Herder JL (2010) The ability of underactuated hands to grasp and hold objects. Mech Mach Theory 45:408-425

Kragten GA, van der Helm FCT, Herder JL (2011) A planar geometric design approach for a large grasp range in underactuated hands. Mech Mach Theory 46:1121-1136

Landsmeer JM (1961) Studies in the anatomy of articulation. I. the equilibrium of the "intercalated" bone. Acta Morphol Neerl Scand 3:287-303

Leardini A, O'Connor J, Catani F, Giannini S (1999) A geometric model of the human ankle joint. J Biomech 32(6):585-591

Leijnse JNAL (1998) A method and device for measuring force transfers between the deep flexors in the musician's hand. J Biomech 31(9):773-779

Li ZM (2002) The influence of wrist position on individual finger forces during forceful grip. J Hand Surg Am 27:886-96

Long C, Conrad PW, Hall EA, Furler SL (1970) Intrinsic-extrinsic muscle control of the hand in power grip and precision handling. an electromyographic study. J Bone Joint Surg Am 52:853-867 
Marguardt E (1965) The Heidelberg pneumatic arm prosthesis. J Bone Joint Surg 47:425-434

Metcalf C, Notley S, Chappell P, Burridge J et al (2008) Validation and application of a computational model for wrist and hand movements using surface markers. IEEE Trans Biomed Eng 55(5):1199-2010

Miyata N, Kouchi M, Kurihaya T, Mochimaru M (2004) Modeling of human hand link structure from optical motion capture data. In: Proceedings of 2004 IEEE/RSJ international conference on intelligent robots and Systems, San Jose

Miyata N, Kouchi M, Mochimaru M, Kurihaya T (2005) Finger joint kinematics from MR images. In: IEEE/RSJ international conference on intelligent robots and systems, Edmonton

Napier J (1993) Hands (Princeton science libary). Princeton University Press, Princeton

Raphael G, Tsianos GA, Loeb GE (2010) Spinal-like regulator facilitates control of a two-degree-of-freedom wrist. J Neurosci 30(28):9431-9444

Ryu JH, Miyata N, Kouchi M, Mochimaru M et al (2003) Analysis of skin movements with respect to bone motions using MR images. Int J CAD/CAM 3:61-66

Salisbury K (1988) Issues in human/computer control of dexterous remote hands. IEEE Trans Aerosp Electron Syst 24(5):591-596

Salisbury K (1991) The Salisbury hand. Ind Robot 18(4):25-26

Sancho-Bru J, Pérez-González A, Vergara-Monedero M, Giurintano D (2001) A 3-D dynamic model of human finger for studying free movements. Jo Biomech 34(11):1491-1500

Santello M, Flanders M, Soechting JF (1998) Postural hand synergies for tool use. J Neurosci 18(23):10105-10115

Schmitt S (2003) Modellierung und simulation biomechanischer Vorgänge am Beispiel Skisprung. Diplomarbeit, Universität Stuttgart

Seo N, Rymer W, Kamper D (2010) Altered digit force direction during pinch grip following stroke. Exp Brain Res 202:891-901

Shinoda Y, Yamaguchi T, Futami T (1986) Multiple axon collaterals of single corticospinal axons in the cat spinal cord. J Neurophysiol 55(3):425-448

Shinoda Y, Yokota JI, Futami T (1981) Divergent projection of individual corticospinal axons to motoneurons of multiple muscles in the monkey. Neurosci Lett 23(1):7-12

Smith-Bindman R, Lipson J, Marcus R, Kim KP et al (2009) Radiation dose associated with common computed tomography examinations and the associated lifetime attributable risk of cancer. Arch Intern Med 22:2078-2086

Snijders CJ, Volkers AC, Mechelse K, Vleeming A (1987) Provocation of epicondylalgia lateralis (tennis elbow) by power grip or pinching. Med Sci Sports Exerc 19:518-523

Stillfried G, Hillenbrand U, Settles M, van der Smagt P (2012) The human hand-a source of inspiration for robotic hands, Springer Tracts on Advanced Robotics, chap. MRI-based skeletal hand movement model (in press)
Stillfried G, van der Smagt P (2010) Movement model of a human hand based on magnetic resonance imaging (MRI). In: 1st international conference on applied bionics and biomechanics, Venice

Takei T, Seki K (2010) Spinal interneurons facilitate coactivation of hand muscles during a precision grip task in monkeys. J Neurosci 30(50):17041-17050

Valero-Cuevas FJ, Laboratory NB (2000) Applying principles of robotics to understand the biomechanics, neuromuscular control and clinical rehabilitation of human digits. In: Proceedings of IEEE international conference on robotics and automation, San Francisco, pp 270-275

Valero-Cuevas FJ, Zajac FE, Burgar CG (1998) Large index-fingertip forces are produced by subject-independent patterns of muscle excitation. J Biomech 31:693-703

van der Helm F (1994) A finite element musculoskeletal model of the shoulder mechanism. J Biomech 27(5):551-569

van der Smagt P, Stillfried G (2008) Using MRI data to compute a hand kinematic model. In: 9th conference on motion and vibration control (MOVIC), München, Germany

van Nierop OA, van der Helm A, Overbeeke KJ, Djajadiningrat TJ (2008) A natural human hand model. Visual Comput 24: $31-44$

Waldron K, Schmiedeler J (2008) Kinematics. In: Siciliano K (ed) Handbook of robotics. Springer, Berlin, pp 9-34

Wilkinson DD, Vandeweghe JM, Matsuoka Y (2003) An extensor mechanism for an anatomical robotic hand. In: Proceedings of the 2003 IEEE International Conference on Robotics and Automation (ICRA '03), Washington, vol 1, pp 238-243

Wilms E, Nader L (1951) Die Technik der Vaduzer Hand. Orthopädie Technik 2(7):7

Woltring H, Lange Ad, Kauer J, Huiskes H (1987) Instantaneous helical axis estimation via natural, cross-validated splines. In: Biomechanics: basic and applied research. Springer, Berlin

Wu G, van der Helm CT, Veeger HEJ et al (2005) ISB recommendation of joint coordinate systems of various joints for the reporting of human joint motion - part ii: shoulder, elbow, wrist and hand. J Biomech 38:981-992

Wu JZ, An KN, Cutlip RG, Andrew ME et al (2009) Modeling of the muscle/tendon excursions and moment arms in the thumb using the commercial software anybody. J Biomech 42:383-388

Wu JZ, An KN, Cutlip RG, Dong RG (2010) A practical biomechanical model of the index finger simulating the kinematics of the muscle/tendon excursions. Bio-Med Mater Eng 20:89-97

Youm Y, Gillespie T, Flatt A, Sprague B (1978) Kinematic investigation of normal mcp joint. J Biomech 11(3):109-118

Zancolli (1979) Structural and dynamic bases of hand surgery. Lippincot, Philadelphia

Zhang X, Sang-Wook L, Braido P (2003) Determining finger segmental centers of rotation in flexion-extension based on surface marker measurement. J Biomech 36:1097-1102 